\title{
SPOROTRICHOID ATOPIC PRURIGO. A COMMON CONDITION WITH AN UNUSUAL CLINICAL PRESENTATION
}

\author{
by Beatriz Di Martino Ortiz, Liz Lezcano, Mirtha Rodríguez Masi, Oilda Knopfelmacher,
} Lourdes Bolla de Lezcano

comment:

Dr. Soe Win Oo

Prurigo is a very common dermatosis with no racial nor sex preference. It is frequently encountered in patients with atopic phenomenon. The causes of prurigo are mainly hypersensitivity reaction, inflammation and infection. Prurigo can also be classified simply as acute or chronic prurigo. The latter is more common type and one of its variant is actinic prurigo. Actinic prurigo is quite common in temperate regions. There are chronic recurrent prurigo nodules on sun-exposed areas of the body such as nape and sides of the neck, backs of the hands, extensor forearms and upper chest, and is usually resistant to treatment. Sporotrichoid atopic prurigo is a rare variant where itchy prurigo nodules are seen along the lymphatic or vascular drainage area, and usually seen in lower limbs. Sporotrichoid atopic prurigo is commonly seen in atopic patients who do hard works and are prone to minor trauma. This includes military persons, people who work in paddy fields and manual labor. The case presented by Beatriz Di Martino Ortiz et al is an unusual type of sporotrichoid atopic prurigo affecting the left axilla. Its cause seems to be inflammation caused by infection. So it is rapidly resolved by systemic antibiotics.

\section{Correspondence:}

Dr. Soe Win Oo

MBBS, MMedSc(Dermatology)

Consultant Dermatologist

Yangon General Hospital

Yangon, Myanmar

E-mai: soewinoo@gmail.com 